\title{
Time-Domain Envelope Measurements for Characterization and Behavioral Modeling of Nonlinear Devices With Memory
}

\author{
François Macraigne, Tibault Reveyrand, Guillaume Neveux, Denis Barataud, \\ Jean-Michel Nebus, Arnaud Soury, and Edouard NGoya
}

\section{Published in}

IEEE Transaction on Microwave Theory and Techniques, August 2006, Volume 54, No 8, pp. 3219-3226

(C) 2006 IEEE Personal use of this material is permitted. However, permission to reprint/republish or redistribute this material for advertising or promotional purposes or for creating new collective works for resale or redistribution to servers or lists, or to reuse any copyrighted component of this work in other works must be obtained from the IEEE. 


\title{
Time-Domain Envelope Measurements for Characterization and Behavioral Modeling of Nonlinear Devices With Memory
}

\author{
François Macraigne, Tibault Reveyrand, Guillaume Neveux, Denis Barataud, Jean-Michel Nebus, \\ Arnaud Soury, and Edouard NGoya
}

\begin{abstract}
This paper presents a calibrated four-channel measurement system for the characterization of nonlinear RF devices such as power amplifiers. The main goal of this study is to perform the characterization of the bandpass response of a nonlinear device-under-test (DUT) driven by modulated carriers. The proposed setup enables the generation of $L$ - or $S$-band (1-4 GHz) carriers with a modulation bandwidth up to $100 \mathrm{MHz}$. The carrier harmonics generated by the nonlinear DUT are ignored and considered to be sufficiently filtered. This characterization setup enables calibrated time-domain measurements of the complex envelopes of both incoming and outgoing $R F$ waves at the input and output of the DUT. This means that the fundamental and harmonic frequencies of the envelope are measured and processed. A large set of modulation formats can be generated by using a computer-controlled arbitrary waveform generator. Complex envelopes are measured by using a four-channel sampling scope. The proposed calibrated setup can be used to study or to validate linearization techniques of power amplifiers. This characterization tool is also well suited for the extraction and validation of behavioral bilateral models of nonlinear RF analog equipment exhibiting memory effects.
\end{abstract}

Index Terms-Behavioral model, calibration, linearity, third-order intermodulation (IM3), time-domain envelope measurements.

\section{INTRODUCTION}

$\mathbf{O}$ NE METHOD for accurate characterization of nonlinear $\mathrm{RF}$ and microwave devices involves use of time-domain measurements. Therefore, absolute magnitude and phase of the spectral components of the signal at the input and output ports of the device-under-test (DUT) must be measured. As a consequence, measurements performed using a vector network analyzer or spectrum analyzer do not provide enough information.

Manuscript received December 15, 2005; revised May 10, 2006. This work was supported in part by the National French Space Agency under Contract 714/CNES/00/8167-IRCOM.

F. Macraigne is with the Institut de Recherche en Communications Optiques et Microondes, University of Limoges, Unité Mixte de Recherche, 87060 Limoges Cedex, France, and also with Anritsu SA9, 91951 Les Ulis Cedex, France (e-mail: francois.macraigne@eu.anritsu.com).

T. Reveyrand, G. Neveux, D. Barataud, J.-M. Nebus, and E. NGoya are with the Institut de Recherche en Communications Optiques et Microondes, University of Limoges, Unité Mixte de Recherche, 87060 Limoges Cedex, France, and also with the Faculté des Sciences et Techniques, XLIM, Unité Mixte de Recherche, Centre National de la Recherche Scientifique, 87060 Limoges Cedex, France (e-mail: tibault.reveyrand@xlim.fr; guillaume.neveux@xlim.fr; denis.barataud@xlim.fr; jean-michel.nebus@xlim.fr; edouard.ngoya@xlim.fr).

A. Soury is with Xpedion Design Systems-24, 87000 Limoges, France (e-mail: arnauds@xpedion.com).

Digital Object Identifier 10.1109/TMTT.2006.879169
A microwave transient analyzer (MTA) and large signal network analyzer (LSNA) enable the extraction of time-domain waveforms at the input and output of nonlinear devices. To get accurate time-domain waveform measurements, the following three main calibration procedures of the LSNA or MTA are required:

- Short open load thru (SOLT), thru-reflect line (TRL), or line-reflect-reflect-match (LRRM) calibration for the correction of wave amplitudes ratios;

- absolute calibration in magnitude by using a power meter;

- absolute phase calibration by using a phase reference generator (this generator is a comb generator with well-known phase relationships between harmonic components of a continuous wave (CW) RF carrier [1]-[3]).

Time-domain measurement principles and techniques are now well established for $\mathrm{CW}$ carriers and their harmonics [4]-[6]. However, accurate measurements of nonlinear devices driven by wideband modulated carriers are required to build behavioral models because modulated signals are necessary to probe memory effects in nonlinear solid-state RF devices [7]-[9]. Basically, using the current configuration of the LSNA [10], which can be considered as a reference instrument for calibrated measurements of time-domain waveforms, the modulation bandwidth of RF signals that can be measured is limited to $10 \mathrm{MHz}$ [10]. This is due to the fact that the harmonic sub-sampling performed within the LSNA operates at a frequency of $25 \mathrm{MHz}$. Some solutions have been already proposed to perform wider bandwidth measurements, but are still under investigation [11]-[13]. Furthermore, the phase calibration of the LSNA for modulated carrier measurements remains a very difficult task because, to our knowledge, there is not, at the moment, any available solution to provide a wideband multitone phase calibrated reference source. This topic is discussed in [14] and [15].

Previously, a calibrated two-channel time-domain measurement system has been developed for the envelope characterization and behavioral modeling of nonlinear $50-\Omega$ amplifiers [16]. In this paper, we propose a calibrated four-channel time-domain measurement system that enables the measurement of the envelope response of mismatched nonlinear RF devices driven by modulated carriers having a modulation bandwidth as high as $100 \mathrm{MHz}$. In its current configuration, the setup operates at $L$ and $S$-bands ( $1-4 \mathrm{GHz}$ ). In this paper, we will focus on the bandpass response of the DUT dedicated to the extraction of behavioral models, taking into account memory effects. The purpose of such models is to be implemented in system-level simulation 


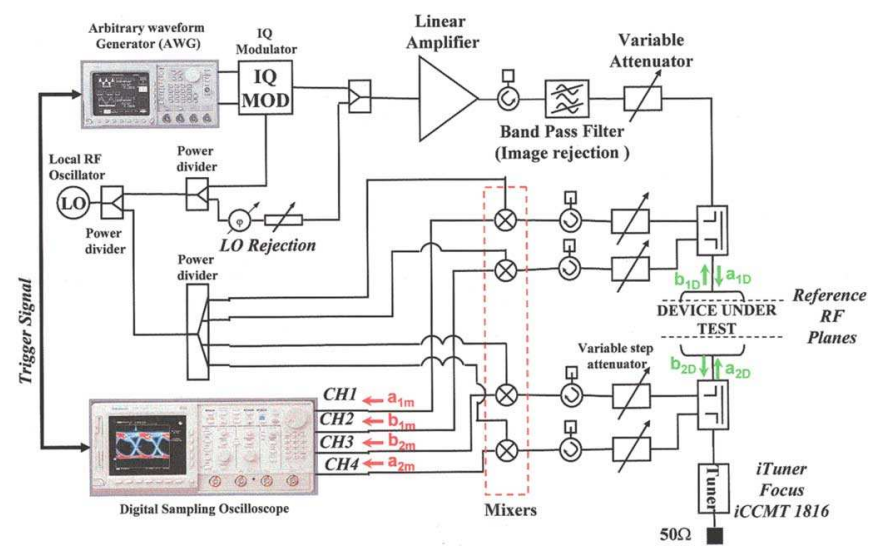

Fig. 1. Time-domain envelope measurement setup. (Color version available online at: http://ieeexplore.ieee.org.)

software like MATLAB/Simulink or Agilent/Ptolemy. Therefore, we will ignore the carrier harmonics and consider that they will be sufficiently filtered out. For that purpose, we have built a four-channel time-domain envelope measurement setup. This setup enables the characterization of the DUT in-band nonlinearities, as well as memory effects and impedance mismatches. The setup is based on the use of a 2-Gigasamples per second (GS/s)-8-bit digital sampling scope (DSO) that directly samples the complex envelopes of the incoming and outcoming RF fundamental carriers at the ports of the DUT. As mentioned in Section V, more powerful sampling scopes can be used without significantly modifying the principle of our study. The measurement setup is presented in Section II. The calibration procedure is explained in Section III. In Section IV-A, the application of the setup to the study of a possible linearization technique of a power transistor is shown. Finally, the application of our characterization tool to the behavioral modeling of a power amplifier is presented in Section IV-B.

\section{Description OF THE MEASUREMENT SETUP}

The block diagram of the measurement setup is given in Fig. 1.

Modulation schemes are achieved by using a computer-controlled arbitrary waveform generator (AWG) (12 bit-250 MS/s). The AWG generates a modulated signal at an IF. This IF signal is then up-converted to $L$ - or $S$-band by using a local oscillator (LO) and an in-phase/quadrature (I/Q) modulator. A specific arrangement, which consists of power dividers, a phase shifter, and a variable attenuator, is used to minimize the LO leakage at the RF output of the I/Q modulator. The microwave modulated signal is then amplified using a very linear amplifier. A bandpass filter is used to reject the signal present at the image frequency. A programmable step attenuator is used to enable power sweeps at the input of the DUT.

The four measurement channels are built with two bi-directional couplers connected at the input and output of the DUT, calibrated step attenuators, isolators, mixers and a four-channel DSO (2 GS/s, 8 bit).

A tuner is also used to make load-pull measurements. This setup must be calibrated in order to get error corrected time-

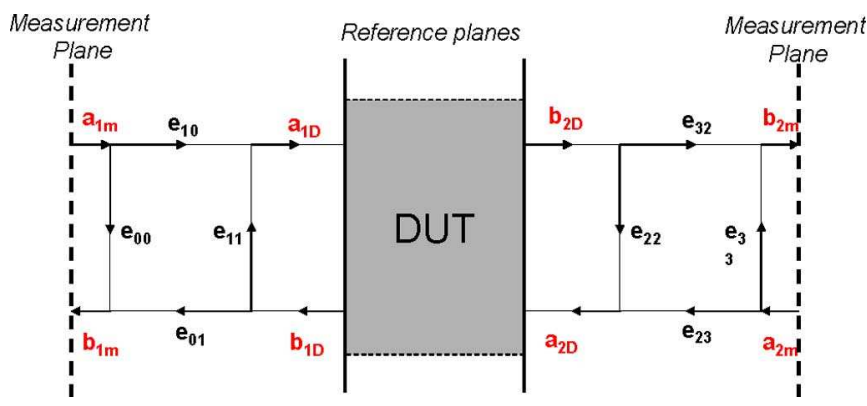

Fig. 2. Flowgraph used for the receiver section calibration procedure. (Color version available online at: http://ieeexplore.ieee.org.)

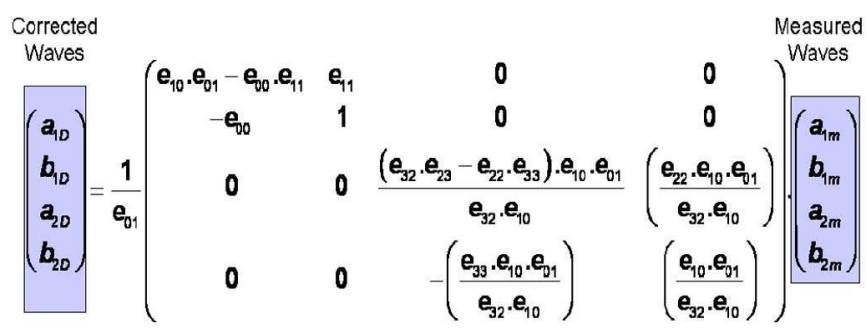

Fig. 3. Error coefficient matrix. (Color version available online at: http://ieeexplore.ieee.org.)

domain envelopes of the incident and reflected RF modulated signals at the DUT reference planes

\section{Description of the CALibration Procedure}

\section{A. Receiver Section Calibration Procedure}

The calibration procedure of the receiver section of the setup consists of determining the frequency response of the four measurement channels at the envelope frequencies of the modulated signal that will be used for the DUT characterization.

The flow-graph representation linking measured wave amplitudes and wave amplitudes at the DUT reference planes is given in Fig. 2.

The associated error coefficient matrix is given in Fig. 3 [17].

In a first step, all the terms of this matrix, except the multiplying term $1 / e_{01}$, are determined using a SOLT, TRL, or LRRM calibration procedure. For that, a CW source is connected to the setup, as shown in Fig. 4.

Calibration standards are connected to the DUT reference planes. The frequency of the source is swept over the bandwidth of interest (Basically, a frequency grid covering a $100-\mathrm{MHz}$ bandwidth above the LO frequency).

Error terms are extracted from the measurements of amplitude waves ratios $b_{j m} / a_{i m}$ made by the DSO at the IF: $f_{\mathrm{IF}}=$ $f_{\text {Source }}-f_{\text {LO }}$.

In a second step, an absolute calibration in magnitude is performed to determine the magnitude of $\left|1 / e_{01}\right|$. For that purpose, a power meter is connected to the input reference plane of the DUT, as shown in Fig. 5.

$\left|e_{01}\right|$ is determined by the following:

$$
\left|e_{01}\right|=10^{\left(\frac{10-P_{e(\mathrm{dBm})}}{20}\right)}\left|\left(e_{01} e_{10}-e_{00} e_{11}\right) a_{1 m}+e_{11} b_{1 m}\right| \text {. }
$$




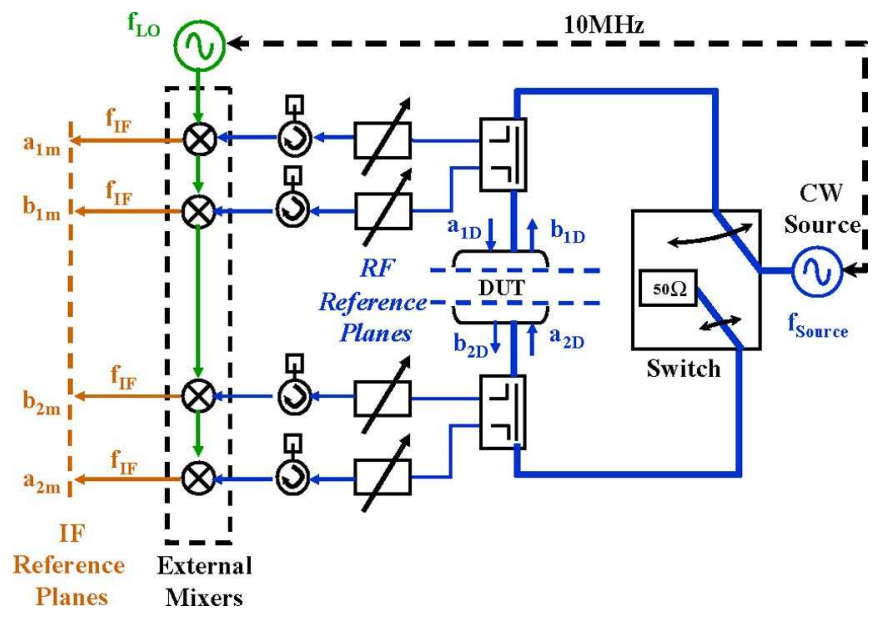

Fig. 4. SOLT, TRL, or LRRM calibration procedure. (Color version available online at: http://ieeexplore.ieee.org.)

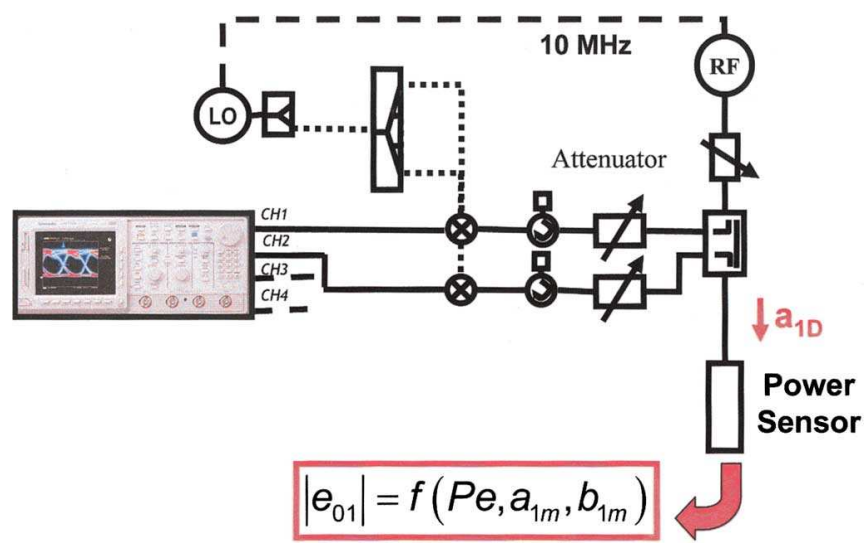

Fig. 5. Absolute power calibration procedure. (Color version available online at: http://ieeexplore.ieee.org.)

$P_{e(\mathrm{dBm})}$ is the power measured by the power meter, while $a_{1 \mathrm{~m}}$ and $b_{1 \mathrm{~m}}$ are the waves measured by channels 1 and 2 of the DSO.

In a third step, we would like to determine the phase of the error term $\angle e_{01}$. Unfortunately, we do not have a multitone RF reference generator with known phase relationships between tones. For that reason, we have to make the assumption that the group delay of the four measurement channels remains constant over the envelope frequency bandwidth of interest. We have validated this assumption by measuring on each channel of the setup a multitone phase aligned signal supplied by a Rhode \& Schwarz generator (SMU 200 A). The signal supplied by this source contained 80 tones covering a $100-\mathrm{MHz}$ bandwidth around a center frequency of $1.6 \mathrm{GHz}$. The phases of the tones were set to $0^{\circ}$. This source was connected in the place of the CW source, as depicted in Fig. 4. A thru connection was made between the reference planes of the DUT. A fast Fourier transform (FFT) was applied to the signal measured by the scope (respectively channels 1 and 3 and then channels 2 and 4). We then plotted the phase of the measured spectral lines versus frequency. We obtained a quasi-perfect line with a negative slope, as depicted in Fig. 6. This indicates that the group delay of the four channels is constant. We observed smooth phase ripples in

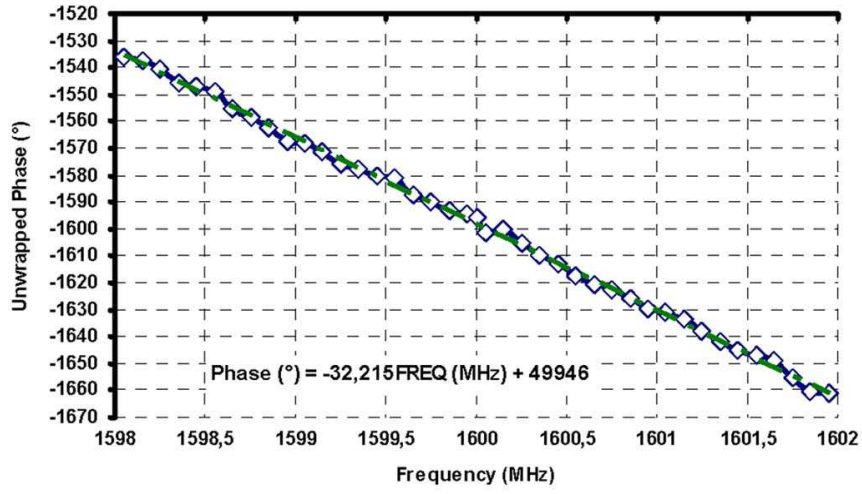

(a)

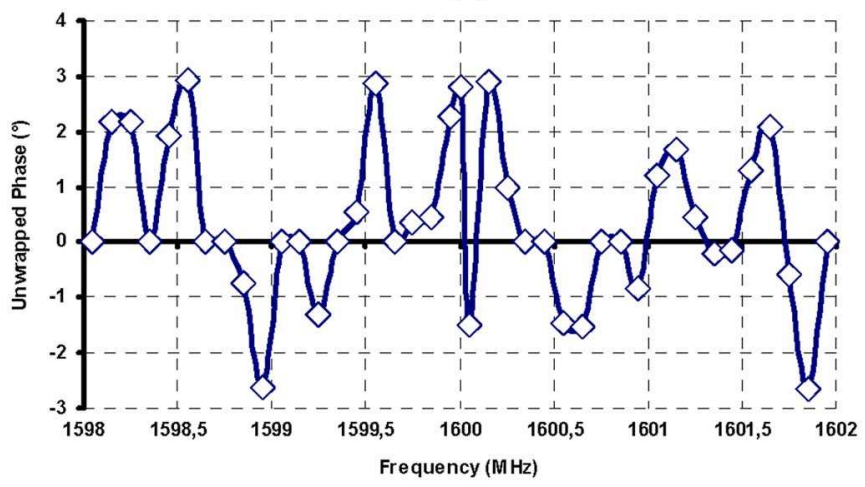

(b)

Fig. 6. Zoom of the unwrapped phase of the multisine measured at channel 1 without: (a) taking into account the time delay and (b) taking into account the time delay. (Color version available online at: http://ieeexplore.ieee.org.)

the order of three degrees, which gives an idea of the accuracy of the assumption.

We also performed this measurement at a center frequency of $3 \mathrm{GHz}$ and came to the same conclusion. By using our calibrated system, as depicted in Fig. 1, we have also measured the four $S$-parameters of linear devices at $L$ - and $S$-band. We have compared the $S$-parameters measured by this setup with $S$-parameters measured with a $360 \mathrm{~B}$ Anritsu vector network analyzer. The differences obtained are in the order of $3^{\circ}$ for phase measurements and $0.1 \mathrm{~dB}$ for magnitude measurements

\section{B. Calibration of the Source Section of the System}

The complex envelope of the signal computed by the personal computer (PC) controlling the whole setup is not exactly the same as the one present in the input RF reference plane of the DUT. This is due to the linear distortion of the source section of the system (from the PC to the input RF reference plane). The chosen envelope model of the source section is represented by a nonideal I/Q modulator, as shown in Fig. 7. The goal of the calibration consists in determining the complex gains $\widetilde{K}_{1}$ and $\widetilde{K}_{2}$ linking the real and imaginary parts of the signal generated by the PC and the real and imaginary parts of the envelope of the RF signal present in the RF reference plane. The determination of these error terms is sketched in Fig. 7.

A specific computed multitone excitation signal calculated by the PC is downloaded into the AWG. This specific signal, noted $s(t)$, is a band-limited multicarrier signal represented by the sum 


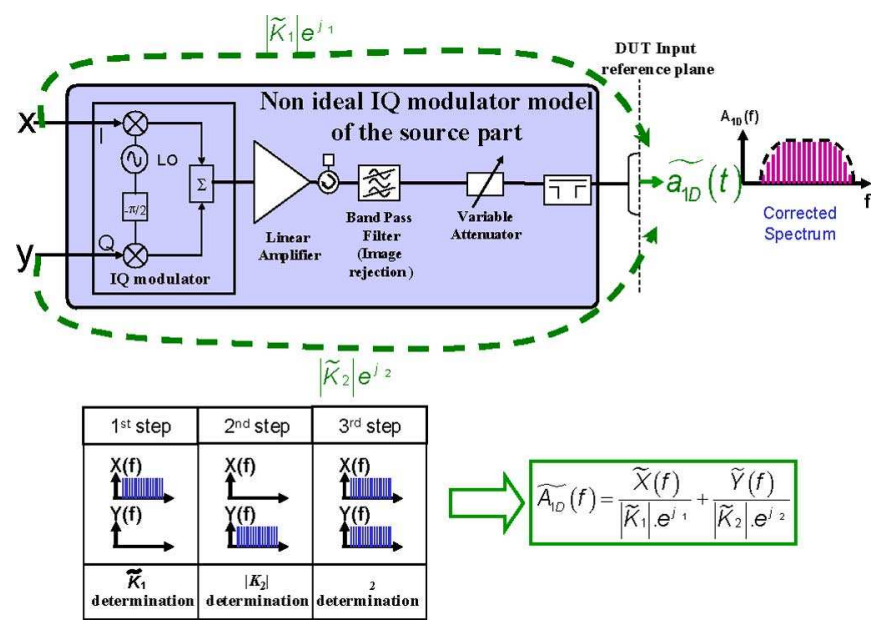

Fig. 7. Calibration of the source section. (Color version available online at: http://ieeexplore.ieee.org.)

of a sufficient number of independent $\mathrm{CW}$ tones with the same magnitude and random relative phases [16]

$$
s(t)=\left[\sum_{n=-N / 2}^{N / 2} A_{n} \cdot e^{j\left(n \Delta \omega t+\varphi_{n}\right)}\right] e^{j\left(\omega_{\mathrm{IF}} t+\varphi_{\mathrm{IF}}\right)} .
$$

The random relative phases $\left\{\varphi_{n}\right\}$ are chosen in order to minimize the peak to average ratio (PAR) of this stimulus (PAR $=2 \mathrm{~dB}$ typically). Using this low PAR signal improves the accuracy of the measurements performed with the scope. We used PAR minimization technique described in [18], but other techniques can be used. Three steps are then required to determine $\widetilde{K}_{1}$ and $\widetilde{K}_{2}$. In a first step, the multitone signal is applied to the $X$ input of the I/Q modulator and no signal is applied to the $Y$ input of the I/Q modulator. The RF envelope $\widetilde{a}_{1 D}$ is determined from DSO measurements $\widetilde{a}_{1 m}, \widetilde{b}_{1 m}$ corresponding, respectively, to channels 1 and 2 via the error correction matrix of Fig. 3. The $\widetilde{K}_{1}$ coefficient is then extracted at each $n \Delta \omega$ frequency component of interest by calculating

$$
\widetilde{K}_{1}=\left(A_{n} / A_{1 D}\right) \cdot e^{j\left(\varphi_{n}-\varphi_{a 1 D}\right)}
$$

where $A_{1 D}$ and $\varphi_{a 1 D}$ are, respectively, the magnitude and phase of each tone of the RF envelope at the DUT reference plane, and $A_{n}$ and $\varphi_{n}$ are the magnitude and phase of the tones generated by the PC. In a second step, the same multitone signal is applied to the $Y$ input of the I/Q modulator and no signal is applied to the $X$ input of the I/Q modulator. The RF envelope $\widetilde{a}_{1 D}$ is determined from DSO measurements. The magnitude of the $\widetilde{K}_{2}$ coefficient is then extracted at each $n \Delta \omega$ frequency component of interest by calculating

$$
\left|\widetilde{K}_{2}\right|=\left(A / A_{1 D}\right)
$$

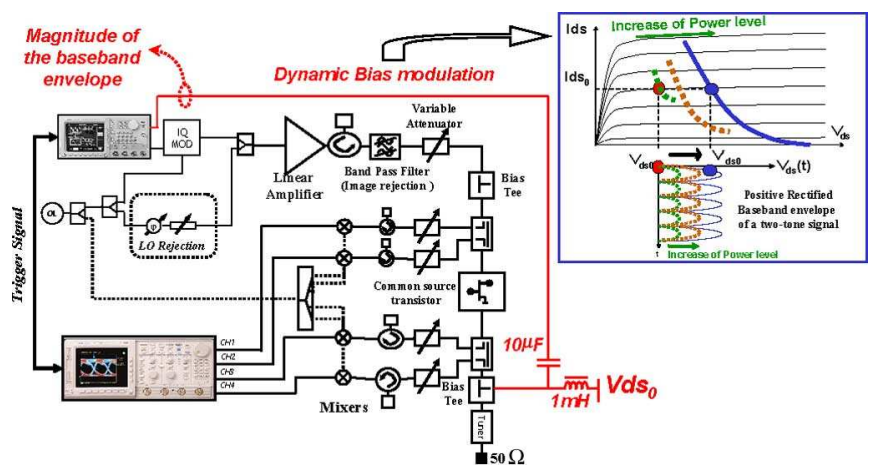

Fig. 8. Dynamic biasing modulation technique used for the linearization of a power transistor (illustrated here for a two-tone RF signal). (Color version available online at: http://ieeexplore.ieee.org.)

The last step enables to characterize the phase $\varphi_{2}$ of $\widetilde{K}_{2}$ by applying $s(t)$ at both $X$ and $Y$ inputs of the I/Q modulator. $\varphi_{2}$ is determined by the following relationship:

$$
\varphi_{2}=-\operatorname{Arc} \cos \left(\frac{|y|^{2}-\left|\widetilde{K}_{1}^{-1} \cdot \widetilde{a}_{1 D}\right|^{2}-\left|\widetilde{K}_{2}^{-1} \cdot \widetilde{a}_{1 D}\right|^{2}}{2 \cdot\left|\widetilde{K}_{1}^{-1} \cdot \widetilde{a}_{1 D}\right| \cdot\left|\widetilde{K}_{2}^{-1} \cdot \widetilde{a}_{1 D}\right|}\right) .
$$

This source section calibration becomes of prime importance if one wants to couple the measurement setup with a system level simulator. In this calibration procedure, the dominant error comes from the 8-bit scope resolution.

\section{Setup Capabilities}

\section{A. Linearization of a Power Transistor}

The calibrated time-domain envelope measurement system has been used to validate a power transistor linearization technique [19]-[21]. It has been applied to a Fujitsu FLK10XM power transistor operating in class $\mathrm{A}(V \mathrm{gs} 0=-1 \mathrm{~V}, I \mathrm{ds} 0=$ $150 \mathrm{~mA}, V \mathrm{ds} 0=5 \mathrm{~V}$ ) at $1.6 \mathrm{GHz}$. First, the load impedance of the transistor has been optimized for maximum output power at a CW carrier frequency of $1.6 \mathrm{GHz}\left(\Gamma \operatorname{load}\left(f_{c}\right)=0.548 e^{j 80^{\circ}}\right)$.

The linearization technique tested here consists of applying to the drain bias circuit of the transistor the magnitude of the envelope of the modulated RF signal that is amplified by the transistor. Therefore, we perform a dynamic biasing technique at the drain port using a network that includes a $10-\mu \mathrm{F}$ capacitor and 1-mH inductor. In the case of a two-tone signal used for intermodulation measurements, the envelope is a sine wave with a frequency equal to the half of the tone spacing $\Delta f$. The magnitude of this envelope is a positive rectified sine wave, as depicted in Fig. 8.

This envelope signal used for drain modulation must be synchronized with the envelope of the modulated signal at the RF input of the transistor. It is achieved by using our setup because we use the two channels of the AWG. One is used to generate the baseband modulation format of the modulated input RF signal. The other one is used to generate the baseband signal for the dynamic drain bias modulation. 

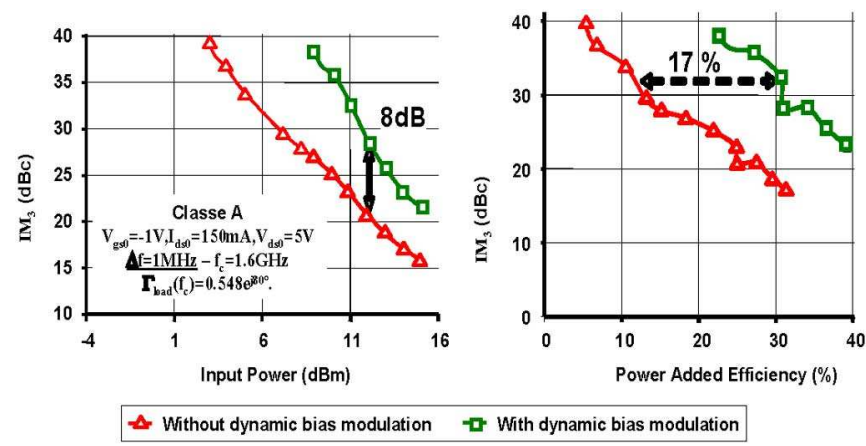

Fig. 9. IM3 versus PAE and versus input power measured with and without dynamic bias modulation. (Color version available online at: http://ieeexplore. ieee.org.)

The principle of the linearity improvement is the following: when the instantaneous input power level of the modulated signal increases, the drain source voltage swing increases. Therefore, the drain source voltage can come close to the ohmic region (low $V_{\mathrm{DS}}$ ), which is a nonlinear region of the $I / V$ characteristics. Thus, intermodulation performance decreases rapidly. By applying a drain bias modulation, the dc quiescent bias point can be pulled away from the ohmic region when the RF input power level increases, as sketched in Fig. 8. Therefore, the nonlinear ohmic region is not reached by the dynamic load line (as shown in Fig. 8), and the linearity of the transistor is enhanced.

We have applied this linearization technique to two kinds of modulated signals: the first one is a two-tone signal with a tone spacing of $1 \mathrm{MHz}$. In this case, the signal used for drain bias modulation is a positive rectified sine wave having a frequency of $0.5 \mathrm{MHz}$. The magnitude and phase of the envelope signal generated for the drain bias modulation are tuned in order to minimize the third-order intermodulation (IM3) at the RF output of the transistor. This tuning is achieved for each input power level driving the device.

Fig. 9 clearly shows the improvements of the linearity performance obtained with the dynamic bias modulation technique.

By using the dynamic bias modulation, the IM3 product increases from 21 to $29 \mathrm{dBc}$ (improvement of $8 \mathrm{~dB}$ ) at an input power of $12 \mathrm{dBm}$. The dynamic bias modulation improves the power-added efficiency (PAE) from $14 \%$ to $31 \%$ for a constant $(C / I)_{3}$ equal to $31 \mathrm{dBc}$. This PAE is calculated without taking into account the consumption of the AWG to generate the drain bias modulation.

The second kind of modulated signals applied to the transistor is a 3.84-Mbit/s quadrature phase-shift keying (QPSK) RF modulated signal. In this case, the signal used for drain-bias modulation has the shape of the magnitude of the baseband modulation format. The magnitude and phase of the envelope signal generated for the drain-bias modulation are tuned in order to minimize the adjacent channel power ratio (ACPR) criterion at the $\mathrm{RF}$ output of the transistor. This tuning is also achieved for each input power level driving the device.

Fig. 10 shows the ACPR improvement obtained when the input RF signal is a QPSK 3.84-Mbit/s modulated signal.

By using the dynamic bias modulation, the ACPR increases from 34 to $44 \mathrm{dBc}$ at an input power of $12 \mathrm{dBm}$.

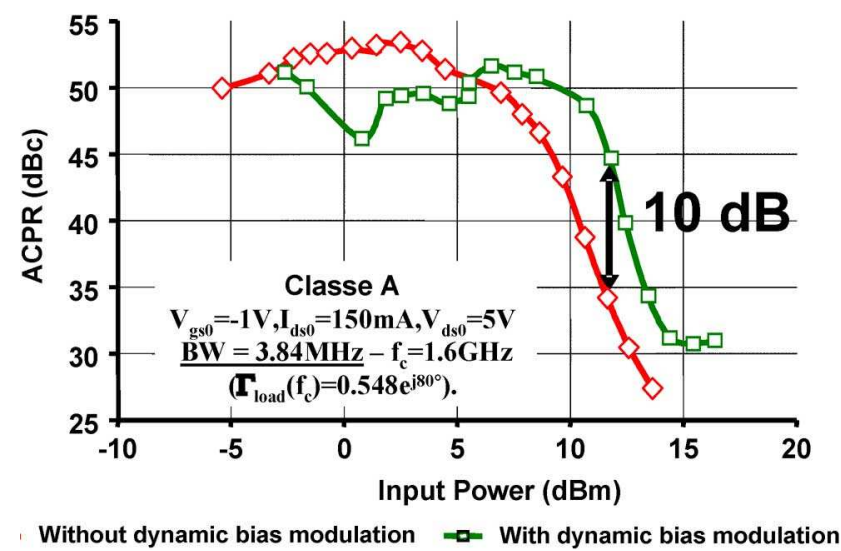

Fig. 10. ACPR versus input power measured with and without dynamic bias modulation. (Color version available online at: http://ieeexplore.ieee.org.)

\section{B. "Black Box" Modeling Application}

An interesting point is that this bench provides information about the complex envelope of the four wave amplitudes present at the access planes of the devices. To exploit this information, we build a black-box model of the device accounted for both memory effects and input/output impedance mismatches. This topic has received increasing attention from the international community since these problems become of prime importance in the design of new wireless communication applications. Among the interesting studies, we can quote the introduction of the "scattering functions" [22]-[25]. Recently, the introduction of the notion of the large-signal $S$-parameters (LSSPs) enabled understanding of these problems in rigorous and powerful circuit envelope formalism [7]. Basically, without entering in the details, we can say that this concept is derived from the Volterra theory and enables us to write, to first order, the input/output relationships as follows:

$$
\begin{aligned}
\hat{b}_{i}(t)= & s_{i}^{\oplus}\left(\left|\hat{a}_{1}(t)\right|\right), \quad i=1,2 \\
& +\frac{1}{2 \pi} \int_{-\infty}^{+\infty} S_{i 1}^{\oplus}\left(\left|\hat{a}_{1}(t)\right|, \Omega\right) \cdot \hat{A}_{1}(\Omega) \cdot e^{j \cdot \Omega \cdot t} \cdot d \Omega \\
& +\frac{1}{2 \pi} \int_{-\infty}^{+\infty} S_{i 1}^{\Delta}\left(\left|\hat{a}_{1}(t)\right|,-\Omega\right) \cdot \frac{\hat{a}_{1}(t)}{\hat{a}_{1}^{*}(t)} \\
& \cdot \hat{A}_{1}^{*}(\Omega) \cdot e^{-j \cdot \Omega \cdot t} \cdot d \Omega \\
& +\frac{1}{2 \pi} \int_{-\infty}^{+\infty} S_{i 2}^{\oplus}\left(\left|\hat{a}_{1}(t)\right|, \Omega\right) \cdot \hat{A}_{2}(\Omega) \cdot e^{j \cdot \Omega \cdot t} \cdot d \Omega \\
& +\frac{1}{2 \pi} \int_{-\infty}^{+\infty} S_{i 2}^{\Delta}\left(\left|\hat{a}_{1}(t)\right|,-\Omega\right) \cdot \frac{\hat{a}_{1}(t)}{\hat{a}_{1}^{*}(t)} \\
& \cdot \hat{A}_{2}^{*}(\Omega) \cdot e^{-j \cdot \Omega \cdot t} \cdot d \Omega .
\end{aligned}
$$

In the above, $\hat{a}_{i}(t)$ and $\hat{b}_{i}(t)$ represent, respectively, the incident and reflected wave amplitudes on port $i$. The variables $\hat{A}_{1}(\Omega)$ and $\hat{A}_{2}(\Omega)$ stand for the frequency spectrum of $\hat{a}_{1}(t)$ and $\hat{a}_{2}(t)$, respectively. Defining a time-varying spectrum $\hat{B}_{i}(\cdots)$, we can rewrite (6) so that

$\hat{b}_{i}(t)=\frac{1}{2 \pi} \int_{-\infty}^{+\infty} \hat{B}_{i}\left(\left|\hat{a}_{1}(t)\right|, \Omega\right) \cdot e^{j \cdot \Omega \cdot t} \cdot d \Omega, \quad$ with $i=1,2$

where

$$
\left[\begin{array}{l}
\hat{B}_{1}(\ldots) \\
\hat{B}_{2}(\ldots)
\end{array}\right]=\left[S^{\oplus}\right] \cdot\left[\begin{array}{c}
\hat{A}_{1}(\Omega) \\
\hat{A}_{2}(\Omega)
\end{array}\right]+\left[S^{\Delta}\right] \cdot\left[\begin{array}{c}
\hat{A}_{1}^{*}(-\Omega) \\
\hat{A}_{2}^{*}(-\Omega)
\end{array}\right]
$$




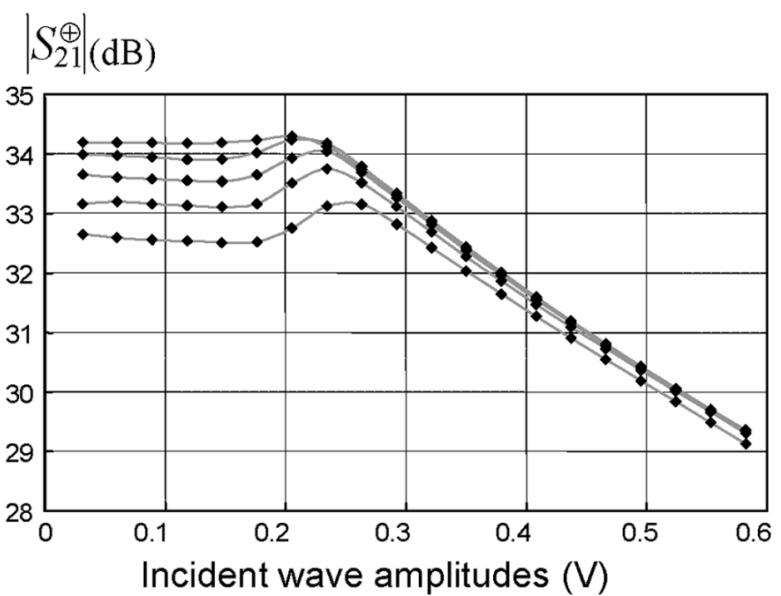

Fig. 11. Input/output transfer characteristic of the amplifier.

with

$$
\left[S^{\oplus}\right]=\left[\begin{array}{l}
s_{1}^{\oplus}\left(\left|\hat{a}_{1}(t)\right|\right)+S_{11}^{\oplus}\left(\left|\hat{a}_{1}(t)\right|, \Omega\right) S_{12}^{\oplus}\left(\left|\hat{a}_{1}(t)\right|, \Omega\right) \\
s_{2}^{\oplus}\left(\left|\hat{a}_{1}(t)\right|\right)+S_{21}^{\oplus}\left(\left|\hat{a}_{1}(t)\right|, \Omega\right) S_{22}^{\oplus}\left(\left|\hat{a}_{1}(t)\right|, \Omega\right)
\end{array}\right]
$$
and

$$
\left[S^{\Delta}\right]=\cdot \frac{\hat{a}_{1}(t)}{\hat{a}_{1}^{*}(t)} \cdot\left[\begin{array}{l}
S_{11}^{\Delta}\left(\left|\hat{a}_{1}(t)\right|, \Omega\right) S_{12}^{\Delta}\left(\left|\hat{a}_{1}(t)\right|, \Omega\right) \\
S_{21}^{\Delta}\left(\left|\hat{a}_{1}(t)\right|, \Omega\right) S_{22}^{\Delta}\left(\left|\hat{a}_{1}(t)\right|, \Omega\right)
\end{array}\right] .
$$

Relation (8) reveals that the sub-system is characterized by two $2 \times 2$ matrices in a similar way that a linear two-port is characterized by only one $2 \times 2$ matrix. It is important to note that the matrix $\left[S^{\Delta}\right]$ vanishes in small-signal conditions $\left(\hat{a}_{1}(t) \rightarrow\right.$ $0)$, and (7) reduces to the classical linear relationship. Thus, the different Volterra kernels in (8) defines the LSSPs, and (7) naturally bridges the extension of the classical $S$-parameters notion towards nonlinear behaviors. As said in [2], the complete identification of the LSSPs can be stated driving the device with a two-tone signal and for three different output terminations. In this paper, we will focus on applications that do not generate low-frequency phenomena or on circuits that do not present spurious long-term memory effects. In such conditions, the influence of the terms $S_{11}^{\Delta}\left(\left|\hat{a}_{1}(t)\right|, \Omega\right)$ and $S_{21}^{\Delta}\left(\left|\hat{a}_{1}(t)\right|, \Omega\right)$ is minor and, thus, can be neglected. Hence, (8) becomes

$$
\left[\begin{array}{l}
\hat{B}_{1}(\ldots) \\
\hat{B}_{2}(\ldots)
\end{array}\right]=\left[S^{\oplus}\right] \cdot\left[\begin{array}{c}
\hat{A}_{1}(\Omega) \\
\hat{A}_{2}(\Omega)
\end{array}\right]+\left[S^{\Delta}\right] \cdot\left[\begin{array}{c}
\hat{A}_{1}^{*}(-\Omega) \\
\hat{A}_{2}^{*}(-\Omega)
\end{array}\right]
$$

with

$$
\left[S^{\oplus}\right]=\left[\begin{array}{l}
s_{1}^{\oplus}\left(\left|\hat{a}_{1}(t)\right|\right)+S_{11}^{\oplus}\left(\left|\hat{a}_{1}(t)\right|, \Omega\right) S_{12}^{\oplus}\left(\left|\hat{a}_{1}(t)\right|, \Omega\right) \\
s_{2}^{\oplus}\left(\left|\hat{a}_{1}(t)\right|\right)+S_{21}^{\oplus}\left(\left|\hat{a}_{1}(t)\right|, \Omega\right) S_{22}^{\oplus}\left(\left|\hat{a}_{1}(t)\right|, \Omega\right)
\end{array}\right]
$$

and

$$
\left[S^{\Delta}\right]=\cdot \frac{\hat{a}_{1}(t)}{\hat{a}_{1}^{*}(t)} \cdot\left[\begin{array}{cc}
0 & S_{12}^{\Delta}\left(\left|\hat{a}_{1}(t)\right|, \Omega\right) \\
0 & S_{22}^{\Delta}\left(\left|\hat{a}_{1}(t)\right|, \Omega\right)
\end{array}\right] .
$$

These considerations simplify the identification procedure. Indeed, for extracting the six terms in (9), we just need to measure the response of the circuit for a single-tone stimulus and for three different output terminations. To fully characterize the circuit, the power and frequency of the single-tone signal have to be swept to cover the frequency bandwidth and power operating range. Fig. 11 presents the LSSPs of a $350-\mathrm{mW} L$-band het-

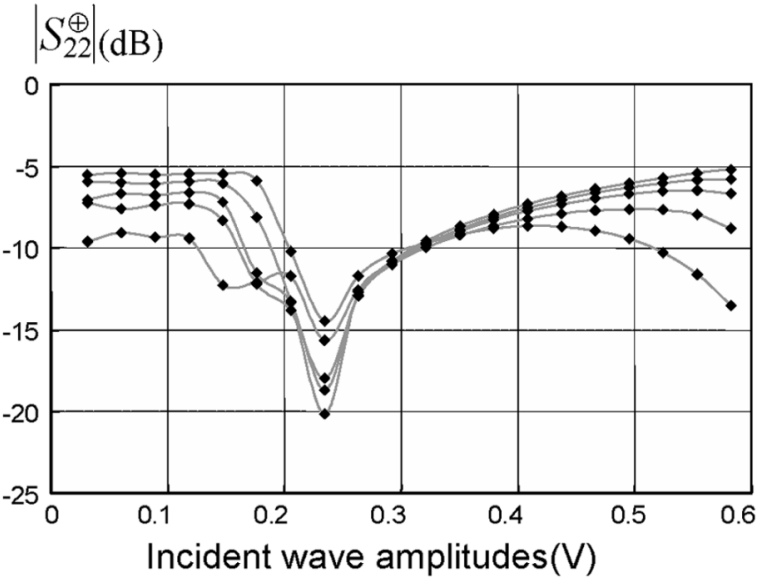

Fig. 12. First output matching characteristic of the amplifier.

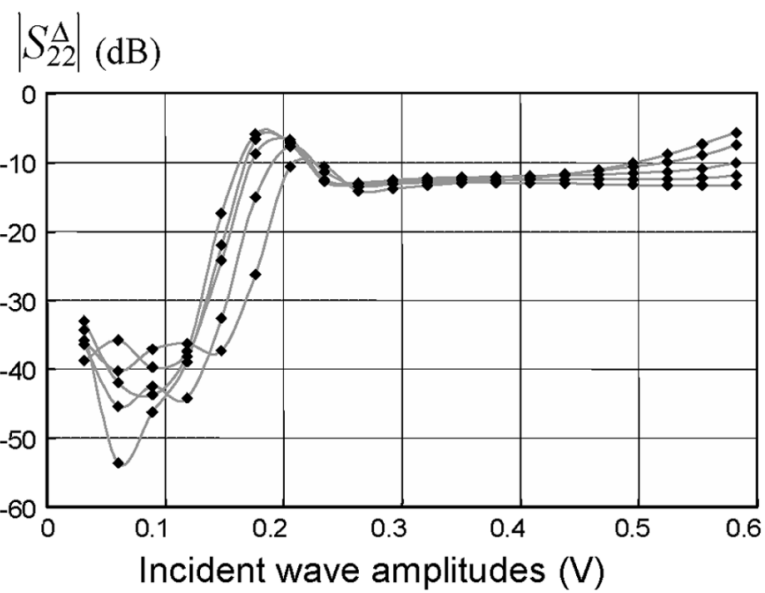

Fig. 13. Second output matching characteristic of the amplifier.

erostructure field-effect transistor (HFET) amplifier extracted for a frequency ranging from -20 to $20 \mathrm{MHz}$ and up to 4-dB compression gain.

Figs. 11-13 illustrate the different transfer characteristics relating the reflected wave amplitude at port 2 to the incident wave amplitudes. We can notably verify that the parameter $S_{22}^{\Delta}$ vanishes at small-signal conditions, exhibiting the fact that (6) reduces to the classical linear relation. It is also interesting to note that the parameter $S_{21}^{\oplus}$ corresponds to the characteristic used for building a model that does not take into account the output impedance mismatch (unilateral model).

Equation (6) has been implemented in the circuit simulator GoldenGate [26] using a specific frequency-domain description (FDD) enabling the handling of nonlinear elements with memory in harmonic-balance (HB) or envelope-transient (ET) engines. Results of a single-tone analysis are presented in Fig. 14 where the results obtained by the model and measurements of the amplifier are superimposed. We can see that the model is able to predict the influence of the variations of the output impedance on the behavior and performance of the amplifier. 

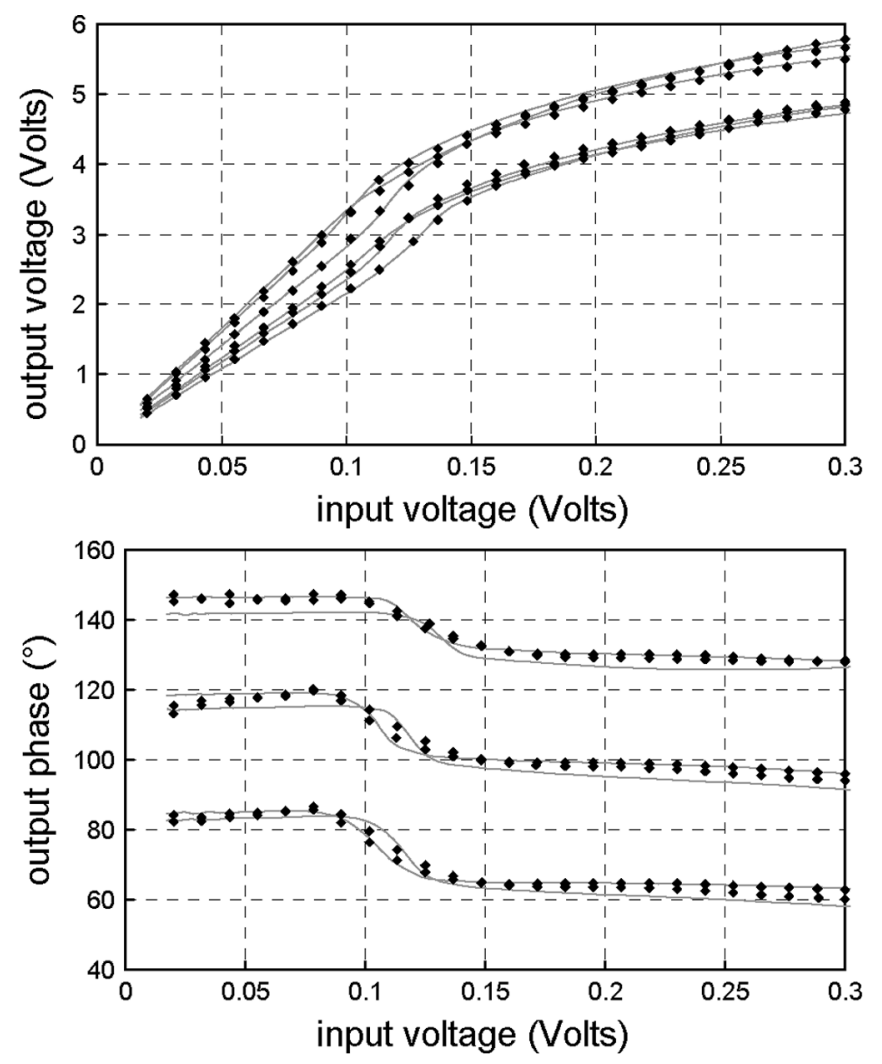

Fig. 14. Performance of the amplifier for a single-tone signal predicted by the model (dots) and measured (lines) for two different output impedances (output reflection coefficient $=0$ and 0.2 ) and for three input frequencies (freq $=-20 \mathrm{MHz}, 0 \mathrm{MHz}$, and $20 \mathrm{MHz}$ ).

\section{CONCLUSION}

A calibrated four-channel time-domain envelope measurement system for the characterization of nonlinear devices such as power amplifiers has been presented. It enables the measurement of the complex envelope of both the incoming and outgoing RF carriers at the input and output of a DUT. The load impedance of the DUT can be controlled by using electromechanical tuners. Two main capabilities of the setup have been presented: the first one consists of applying a linearization technique to improve the linearity performance of a MESFET transistor. The performance of the transistor has been clearly improved by applying a dynamic drain bias modulation. The second main capability of this characterization tool is the validation or the extraction of behavioral bilateral models of nonlinear devices exhibiting memory effects. The behavioral model of an $L$-band medium power amplifier has been extracted thanks to this new characterization tool and the LSSP's formalism. The performance of the amplifier for a single-tone signal predicted by the model and measured for two different output impedances shows very good agreement and illustrates the interest in such a characterization tool for the system-level modeling of nonlinear devices. An interesting extension of the setup can be made by using a $40-\mathrm{GS} / \mathrm{s}-15-\mathrm{GHz}$ sampling scope. This will eliminate the mixer stages of our measurement channels. A next step in the behavioral modeling will consist of taking into account both mismatch effects and long-term memory effects. Another important feature that is now under investigations is the experimental and theoretical study of linearization of power amplifiers using baseband predistortion techniques. For that, both calibrated measurements and behavioral models like those proposed in this paper are required.

\section{ACKNOWLEDGMENT}

The authors want to thank A. Mallet and F. Gizard, both with the National French Space Agency, Toulouse, France, for their helpful assistance.

\section{REFERENCES}

[1] J. Verspecht and K. Rush, "Individual characterization of broadband sampling oscilloscopes with a "nose to nose" calibration procedure," IEEE Trans. Microw. Instrum. Meas., vol. 43, no. 2, pp. 347-354, Apr. 1994.

[2] J. Verspecht, "Calibration of a measurement system for high frequency nonlinear devices," Ph.D. dissertation, Dept. Electron., Vrije Univ. Brussel, Brussels, Belgium, Sep. 1995.

[3] U. Lott, "Measurement of magnitude and phase of harmonics generated in nonlinear microwave two-ports," IEEE Trans. Microw. Theory Tech., vol. 37, no. 10, pp. 1506-1511, Oct. 1989.

[4] D. Barataud, A. Mallet, M. Campovecchio, J. M. Nebus, J. P. Villotte, and J. Verspecht, "Measurements of time domain voltage/current waveforms at R.F. and microwave frequencies for the characterization of nonlinear devices," in IEEE Instrum. Meas. Technol. Conf., St. Paul, MN, 1998, pp. 1006-1010.

[5] G. Kompa and F. van Raay, "Error-corrected large-signal waveform measurement system combining network analyzer and sampling oscilloscope capabilities," IEEE Trans. Microw. Theory Tech., vol. 38, no. 4, pp. 358-365, Apr. 1990.

[6] P. J. Tasker, "Non-linear vector network analyser (NLVNA)," in 14th IEE Microw. Meas. Training Course Conf., May 9-13, 2005, pp. $15-15 / 19,2005 / 10870$.

[7] A. Soury, E. Ngoya, and J. Rousset, "Behavioral modeling of RF and microwave circuit blocs for hierarchical simulation of modern transceivers," in IEEE MTT-S Int. Microw. Symp. Dig., Long Beach, CA, Jun. 2005, [CD ROM]

[8] C. J. Clark, G. Chrisikos, M. S. Muha, A. A. Moulthrop, and C. P. Silva, "Time-domain envelope measurement technique with application to wideband power amplifier modeling," IEEE Trans. Microw. Theory Tech., vol. 46, no. 12, pp. 2531-2540, Dec. 1998.

[9] R. Hajji, F. Beauregard, and F. M. Ghannouchi, "Multitone power and intermodulation load-pull characterization of microwave transistors suitable for linear SSPA's design," IEEE Trans. Microw. Theory Tech. vol. 45, no. 7, pp. 1093-1099, Jul. 1997.

[10] M. Vanden Bossche, "Theoretical background on LSNA technology," LSNA Technol. Library NMDG bvba, Brussels, Belgium, 1989 [Online]. Available: Available: http://www.nmdg.be

[11] W. Van Moer and Y. Rolain, "An improved broadband conversion scheme for the large signal network analyzer," in IEEE MTT-S Int. Microw. Symp. Dig., Long Beach, CA, Jun. 2005, pp. 1501-1504.

[12] K. A. Remley, D. M. M.-P. Schreurs, D. F. Williams, and J. Wood, "Extended NVNA bandwidth for long-term memory measurements," in IEEE MTT-S Int. Microw. Symp. Dig., Fort Worth, TX, Jun. 2004, vol. 3, pp. 1739-1742.

[13] D. Schreurs and K. Remley, "Bandwidth extension using stitching approach," in ARFTG Nonlinear Meas. Workshop, Washington, DC, Nov. 30, 2005, 6 pp.

[14] S. Vandenplas, J. Verspecht, F. Verbeyst, E. Vandamme, and M. V. Bossche, "Calibration issues for the large signal network analyzer (LSNA)," in 60th ARFTG Conf. Dig., Washington, DC, Dec. 5-6, 2002, pp. 99-106.

[15] K. A. Remley, P. D. Hale, D. I. Bergman, and D. Keenan, "Comparison of multisine measurements from instrumentation capable of nonlinear system characterization," in 66th ARFTG Conf. Dig., Washington, DC, Dec. 2005, pp. 34-43.

[16] T. Reveyrand, C. Maziere, J. M. Nébus, R. Quéré, A. Mallet, L. Lapierre, and J. Sombrin, "A calibrated time domain envelope measurement system for the behavioral modeling of power amplifiers," in Eur. Microw. Week GaAs 2002, Milan, Italy, Sep. 2002, pp. 237-240.

[17] J. V. Butler, Rytting, K. Douglas, M. F. Iskander, F. Magdy, R. D. Pollard, and M. Vanden Bossche, "16-term error model and calibration procedure for on-wafer network analysis measurements," IEEE Trans. Microw. Theory Tech., vol. 39, no. 12, pp. 2211-2221, Dec. 1991.

[18] P. Guillaume, J. Schoukens, R. Pintelon, and I. Kollar, "Crest factor minimization using nonlinear Chebyshev approximation methods," IEEE Trans. Microw. Instrum. Meas., vol. 40, no. 6, pp. 982-989, Jun. 1991. 
[19] C. Duvanaud, F. Robin, S. Dardenne, F. Huin, and L. Dascalescu, "Effects of low-frequency drain termination and injection on nonlinear amplifier performances," Int. J. RF Microw. Comput.-Aided Eng., vol. 15, no. 2, pp. 231-240, 2005.

[20] A. Katz, "Linearization: Reducing distortion in power amplifiers," IEEE Micro, pp. 37-39, Dec. 2001.

[21] P. M. Asbeck, T. Itoh, Y. Qian, M. F. Chang, L. Milstein, G. Hanington, P. F. Chen, V. Schultz, D. W. Lee, and J. Arun, "Device and circuit approaches for improved linearity and efficiency in microwave transmitters," in IEEE MTT-S Int. Microw. Symp. Dig., Denver, CO, Jun. 1998, pp. 327-330.

[22] J. Verspecht, "Scattering functions for nonlinear behavioral modeling in the frequency domain," in IEEE MTT-S Int. Microw. Symp. Fundamentals Nonlinear Behavioral Modeling: Foundations Applicat. Workshop, Philadelphia, Jun. 2003, [CD ROM].

[23] J. A. Jargon, K. C. Gupta, and D. C. DeGroot, "Nonlinear large-signal scattering parameters: Theory and applications," in ARFTG Conf. Dig., Jun. 2004, pp. 157-174.

[24] J. Verspecht, D. E. Root, J. Wood, and A. Cognata, "Broad-band multiharmonic frequency domain behavioral models from automated large signal vectorial network measurements," in IEEE MTT-S Int. Microw. Symp. Microw. Symp. Fundamentals Nonlinear Behavioral Modeling: Foundations Applicat. Workshop, Long Beach, CA, Jun. 2005, [CD ROM].

[25] D. E. Root, J. Verspecht, D. Sharrit, J. Wood, and A. Cognata, "Broad-band poly-harmonic distortion (PHD) behavioral models from fast automated simulations and large-signal vectorial network measurements," IEEE Trans. Microw. Theory Tech., vol. 53, no. 11, pp. 3656-3664, Nov. 2005.

[26] “GoldenGate Users Manual," Xpedion Des. Syst., Santa Clara, 2005. [Online]. Available: Available: http://www.xpedion.com/

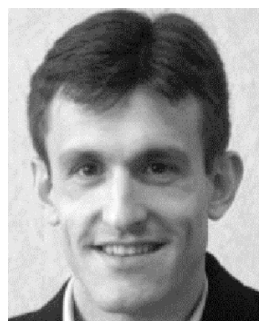

François Macraigne was born in Limoges, France, on November 6, 1977. He received the Ph.D. degree in electronics from the University of Limoges, Limoges, France, in 2005.

$\mathrm{He}$ is currently a Sales Engineer with Anritsu SA9, Les Ulis, France.

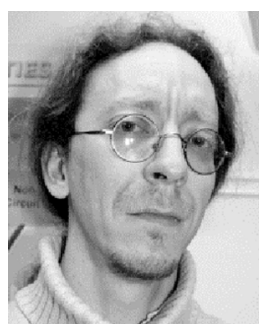

Tibault Reveyrand was born in Paris, France, on September 20, 1974. He received the Ph.D. degree from the University of Limoges, Limoges, France, in 2002.

From 2002 to 2004, he was a Post-Doctoral Scientist with the Centre National d'Etudes Spatiales (CNES) (French Space Agency). In 2005, he became a Contractual Center National de la Recherche Scientifique (CNRS) Engineer with XLIM [formerly the Institut de Recherche en Communications Optiques et Microondes (IRCOM)], Limoges, France. His research interests include the characterization and modeling of RF and microwave nonlinear components.

Dr. Reveyrand was the recipient of the 2002 European GaAs Best Paper Award.

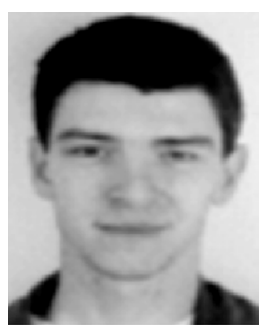

Guillaume Neveux was born in Civray, France, in 1976. He received the Diplôme d'Etudes Approfondies (DEA) degree from the Université Paris 11, Orsay, France, in 2000, and the Ph.D. degree in electronics and communications from the National Superior Institute of Telecommunications (ENST), Paris, France, in 2003.

Since 2004, he has been with the Instrumentation Group, XLIM Laboratory, University of Limoges, Limoges, France. His research interests include nonlinear measurement with LSNA and the study of

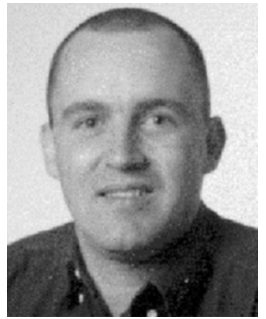

Denis Barataud was born in Saint-Junien, France, in 1970. He graduated from the Ecole Nationale Superieure de Telecommunications de Bretagne, Bretagne, France, in 1994. He received the Ph.D. degree in electronics from the University of Limoges, Limoges, France, in 1998.

From 1998 to 1999, he was a Post-Doctoral Scientist with the Microwave Laboratory, Centre National d'Etudes Spatiales (CNES), Toulouse, France. Since 2000, he has been with the XLIM [formerly the Institut de Recherche en Communications Optiques et Microondes (IRCOM)], University of Limoges, Limoges, France, where he became an Assistant Professor in 2001. His research interests include the development of time-domain equipment and techniques for the characterization of nonlinear devices.

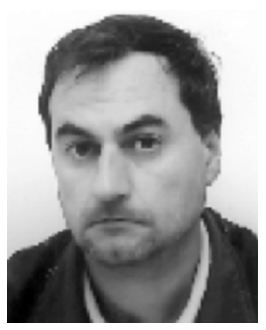

Jean-Michel Nebus was born in Bourganeuf, France in 1963. He received the Ph.D. degree in electronics from the University of Limoges, Limoges, France in 1988.

He was a Project Engineer with ALCATEL Space Industries France. He is currently a Professor with the XLIM [formerly the Institut de Recherche en Communications Optiques et Microondes (IRCOM)], University of Limoges. His main area of interest is nonlinear microwave device characterization and design.

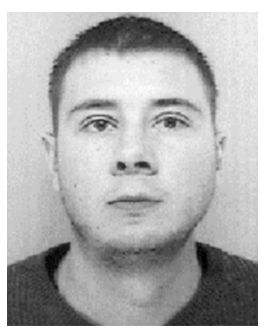

Arnaud Soury was born in Confolens, France, in 1975. He received the Ph.D. degree in electrical engineering from the University of Limoges, Limoges, France, in 2002.

From 2003 to 2004, he was a Post-Doctoral Scientist with the French Centre National de la Recherche Scientifique (CNRS), XLIM [formerly the Institut de Recherche en Communications Optiques et Microondes (IRCOM)], University of Limoges. In 2005, he joined Xpedion Design Systems, Limoges, France, where he is currently with the Development Team involved with the circuit-level simulation tool GoldenGate. His main research interests are in the field of computed-aided design techniques for RF and microwave circuits including behavioral modeling, analysis techniques, and stability of electronic devices.

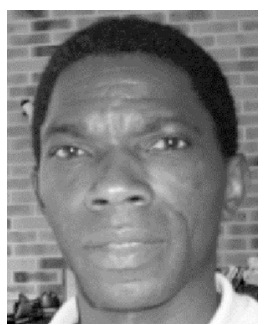

Edouard NGoya received the Ph.D. degree in electronics from the University of Limoges, Limoges, France, in 1988.

In 1990, he joined the French Centre National de la Recherche Scientifique (CNRS), XLIM [formerly the Institut de Recherche en Communications Optiques et Microondes (IRCOM)], University of Limoges. He is the inventor of key analog simulation and modeling technologies such as compressed transient, ET, and dynamic Volterra series. Since 1998, he has been the Consultant Chief Scientist with Xpedion Design Systems, Milpitas, CA. His current research activities focus on modeling and simulation techniques for large-scale integration RF circuits, RF sytem-on-chip (SOC) and system-in-package (SIP). 